\title{
Optimal Power Control for Superimposed Pilots in Uplink Massive MIMO Systems
}

Daniel Verenzuela, Andreas Bergström and Emil Björnson

The self-archived postprint version of this journal article is available at Linköping University Institutional Repository (DiVA):

http://urn.kb.se/resolve?urn=urn:nbn:se:liu:diva-158405

N.B.: When citing this work, cite the original publication.

Verenzuela, D., Bergström, A., Björnson, E., (2018), Optimal Power Control for Superimposed Pilots in Uplink Massive MIMO Systems, 2018 CONFERENCE RECORD OF 52ND ASILOMAR CONFERENCE ON SIGNALS, SYSTEMS, AND COMPUTERS, , 499-503.

https://doi.org/10.1109/ACSSC.2018.8645504

Original publication available at:

https://doi.org/10.1109/ACSSC.2018.8645504

Copyright: Institute of Electrical and Electronics Engineers (IEEE)

http://www.ieee.org/

(C)2018 IEEE. Personal use of this material is permitted. However, permission to reprint/republish this material for advertising or promotional purposes or for creating new collective works for resale or redistribution to servers or lists, or to reuse any copyrighted component of this work in other works must be obtained from the IEEE. 


\title{
Optimal Power Control for Superimposed Pilots in Uplink Massive MIMO Systems
}

\author{
Daniel Verenzuela*, Andreas Bergström*, Emil Björnson*, \\ *Department of Electrical Engineering (ISY), Linköping University, Linköping, Sweden.
}

\begin{abstract}
In Massive MIMO, the pilot contamination effect reduces the spectral efficiency (SE) gains and Superimposed pilot (SP) transmission has been proposed to mitigate this effect. SP is based on transmitting pilot and data symbols simultaneously to allow for longer pilots and no pilot overhead. This work studies the optimal power control strategies in the uplink of a Massive MIMO system with SP and detection based on maximum ratio combining. The optimization objectives are maximum product of SINRs and max-min fairness, and these problems are reformulated as geometric programs which allow for efficient implementations. The numerical results indicate that the SE gains from the optimal power control with respect to the heuristic statistical channel inversion power control, are more significant when the interference from pilot symbols is subtracted before data detection.
\end{abstract}

\section{INTRODUCTION}

Massive MIMO is a key technology for the next generation of wireless communications for its ability to deliver high spectral efficiency (SE) and energy efficiency (EE) [1], [2]. This is achieved by the use of a large number of antennas at the base station (BS) to spatially multiplex several user equipments (UE) on the same time-frequency resource.

In Massive MIMO, channel estimation is of paramount importance in order to spatially separate the UEs by coherent signal processing to harvest the benefits of having a large number of BS antennas. A standard method to estimate the channels between the BS and UEs, is to send pilot signals in the uplink (UL). Since in typical cellular deployments there are more UEs than available pilots signals, these pilots need to be reused among cells giving rise to the pilot contamination effect which degrades the SE [3].

To mitigate the effect of pilot contamination, the works [2], [4]-[6] use the spatial correlation between channels received at different BS antennas, whereas in [1], [7]-[9] the pilot overhead is increased to allow for longer pilot sequences that can be reused less often among cells.

An alternative approach, is the use of superimposed pilot (SP) transmission in which the pilot and data symbols are transmitted simultaneously [10]-[18]. This means, that longer pilot symbols can be used with no pilot overhead. Thus, there are more symbols used for channel estimation and the pilots can be reused far less often among cells [15]-[18]. However, the drawback of using SP is that the channel estimates and the data symbols in the UL become correlated which, in turn, creates additional interference that limits the SE [17], [18].

In [17], closed-form expressions for achievable rates with $\mathrm{SP}$ were found considering maximum ratio combining (MRC) in the UL data detection. It was also shown that the use of SP has the potential to offer high SE and large multiplexing gains if the interference from pilot symbols in the data detection process is perfectly removed. On the other hand, in previous works [15]-[18], the transmission power strategy implemented with SP has been based on suboptimal statistical channel inversion power control.

This article studies the optimal power control strategies with SP and MRC to determine the SE gains that can be obtained with respect to statistical channel inversion power control. The closed-form achievable rates obtained in [17] are used to cast two optimization problems with the following objectives: maximize the product of signal-to-interference plus noise ratios (SINRs) among UEs, and maximize the minimum SINRs among UEs. These optimization problems are then rewritten as geometric programs that can be solve efficiently in polynomial time [2], [19], [20].

\section{SySTEM MODEL}

This paper studies the UL of a multicell Massive MIMO system where each BS has $M$ antennas and serves $K$ singleantenna UEs. The set $\Phi$ encloses the indices of all BSs in the system such that $\mathrm{BS}_{l}$ refers to the BS in cell $l \in \Phi$, and the UE $k \in\{1, \ldots, K\}$ that is served in cell $l$ is denoted as $\mathrm{UE}_{l k}$. The communication channels are modeled as uncorrelated Rayleigh block fading where each channel is considered to be constant and frequency flat over a coherence block of time period $T_{c}$ and bandwidth $B_{c}$, respectively. The total bandwidth $B_{\mathrm{W}}$ is equally divided among all coherence blocks, so that $B_{\mathrm{W}} / B_{c}$ is an integer, and each block contains $\tau_{c}=B_{c} T_{c}$ complex samples. The channel between the $M$ antennas of $\mathrm{BS}_{l}$ and $\mathrm{UE}_{l k}$ is defined as ${ }^{1} \mathbf{h}_{l l k} \sim \mathcal{C N}\left(\mathbf{0}, \beta_{l l k} \mathbf{I}_{M}\right)$ with $\beta_{l l k} \geq 0$ being the large-scale fading coefficient. An SP transmission protocol is assumed as illustrated in Fig. 1, where pilot and data symbols are transmitted simultaneously within the whole coherence block. The transmitted data and pilot symbols from $\mathrm{UE}_{l k}$, in all $\tau_{c}$ samples of the coherence block, are given by $\mathbf{s}_{l k} \in \mathbb{C}^{\tau_{c} \times 1}$, and $\phi_{l k} \in \mathbb{C}^{\tau_{c} \times 1}$, respectively. The transmission powers for data and pilot symbols from $\mathrm{UE}_{l k}$ are defined as $\rho_{l k}$, and $q_{l k}$, respectively. Note that since both pilot and data symbols are transmitted simultaneously, the transmission power needs to be shared between the two such that $\rho_{l k}+q_{l k} \leq P_{\max }$ where $P_{\max }$ is the maximum transmission power per UE. The received signal $\mathbf{Y}_{l} \in \mathbb{C}^{M \times \tau_{c}}$ at $\mathrm{BS}_{l}$ is

\footnotetext{
${ }^{1} \mathrm{CN}(\cdot, \cdot)$ denotes a circularly symmetric complex Gaussian distribution.
} 


$$
\begin{aligned}
D_{l k}= & \underbrace{M \sum_{(\ell, i) \in \mathcal{S}_{l k} \backslash(l, k)} q_{\ell i} \rho_{\ell i} \beta_{l \ell i}^{2}+\frac{M}{\tau_{c}} \sum_{\ell \in \Phi} \sum_{i=1}^{K}\left(a_{\ell i}+\rho_{\ell i}\right) \rho_{\ell i} \beta_{l \ell i}^{2}}_{\text {Coherent interference }} \\
& +\underbrace{\frac{2}{\tau_{c}} \sum_{(\ell, i) \in \mathcal{S}_{l k}} a_{\ell i} \rho_{\ell i} \beta_{l \ell i}^{2}+\frac{1}{\tau_{c}^{2}} \sum_{\ell \in \Phi} \sum_{i=1}^{K} \rho_{\ell i}^{2} \beta_{l \ell i}^{2}+\left(\sum_{(\ell, i) \in \mathcal{S}_{l k}} q_{\ell i} \beta_{l \ell i}+\frac{1}{\tau_{c}} \sum_{\ell \in \Phi} \sum_{i=1}^{K} \rho_{\ell i} \beta_{l \ell i}+\frac{\sigma^{2}}{\tau_{c}}\right)\left(\sum_{\ell \in \Phi} \sum_{i=1}^{K}\left(a_{\ell i}+\rho_{\ell i}\right) \beta_{l \ell i}+\sigma^{2}\right)}_{\text {Non-coherent interference and noise }}
\end{aligned}
$$

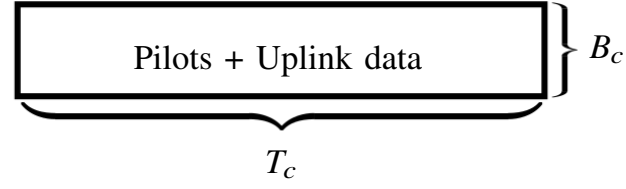

Fig. 1: SP transmission protocol.

$$
\mathbf{Y}_{l}=\sum_{\ell \in \Phi} \sum_{i=1}^{K} \mathbf{h}_{l \ell i}\left(\sqrt{\rho_{\ell i}} \mathbf{s}_{\ell i}^{T}+\sqrt{q_{\ell i}} \boldsymbol{\phi}_{\ell i}^{T}\right)+\mathbf{N}_{l}
$$

where $\mathbf{N}_{l}=\left[\mathbf{n}_{l 1}, \ldots, \mathbf{n}_{l \tau_{c}}\right]$ is the noise matrix with i.i.d. columns distributed as $\mathbf{n}_{l j} \sim \mathcal{C N}\left(\mathbf{0}, \mathbf{I}_{M} \sigma^{2}\right) \forall j \in\left\{1, \ldots, \tau_{c}\right\}$ and the operator $(\cdot)^{T}$ stands for transpose.

\section{Channel Estimation}

To estimate the channels, the standard linear minimum mean squared error (LMMSE) technique is used. Consider a set of $\tau_{c}$ orthogonal pilot sequences of length $\tau_{c}$, denoted as $\mathcal{U}_{\tau_{c}}$, and let $\phi_{l k} \in \mathcal{U}_{\tau_{c}}$, be the pilot sequence assigned to $\mathrm{UE}_{l k}$ with $\left|\left[\boldsymbol{\phi}_{l k}\right]_{j}\right|=1, \forall j \in\left\{1, \ldots, \tau_{c}\right\}$. Each BS assigns $K \leq \tau_{c}$ different pilot sequences to the UEs served in its cell. Since the total number of UEs in a cellular network is usually much larger than $\tau_{c}$, some pilots need to be reused among cells. Thus, the set $\mathcal{S}_{l k}$ contains the indices corresponding to the UEs that use the same pilot as $\mathrm{UE}_{l k}$ (including $\mathrm{UE}_{l k}$ itself), defined as

$$
\mathcal{S}_{l k}=\left\{(\ell, i): \phi_{\ell i}^{H} \phi_{l k} \neq 0\right\}
$$

where the operator $(\cdot)^{H}$ denotes Hermitian transpose. To estimate the channel of $\mathrm{UE}_{l k}, \mathrm{BS}_{l}$ conducts a de-spreading operation by multiplying $\mathbf{Y}_{l}$ with $\boldsymbol{\phi}_{l k}^{*} / \sqrt{\tau_{c}}$, resulting in [17]

$$
\mathbf{z}_{l l k}=\sum_{(\ell, i) \in \mathcal{S}_{l k}} \sqrt{q_{\ell i} \tau_{c}} \mathbf{h}_{l \ell i}+\sum_{\ell \in \Phi i=1}^{K} \sqrt{\frac{\rho_{\ell i}}{\tau_{c}}} \mathbf{h}_{l \ell i} \mathbf{s}_{\ell i}^{T} \boldsymbol{\phi}_{l k}^{*}+\sum_{j=1}^{\tau_{c}} \mathbf{n}_{l j} \frac{\left[\boldsymbol{\phi}_{l k}\right]_{j}^{*}}{\sqrt{\tau_{c}}} .
$$

The signal $\mathbf{z}_{l l k}$ is then used by $\mathrm{BS}_{l}$ to compute $\hat{\mathbf{h}}_{l l k}$ which is the LMMSE estimate of $\mathbf{h}_{l l k}$. This result is summarized in the following lemma.

Lemma 1. The LMMSE estimate of $\mathbf{h}_{l l k}$ is

$$
\hat{\mathbf{h}}_{l l k}=\frac{\gamma_{l l k}}{\sqrt{q_{l k} \tau_{c}}} \mathbf{z}_{l l k}
$$

where

$$
\gamma_{l l k}=\frac{q_{l k} \tau_{c} \beta_{l l k}}{\sum_{(\ell, i) \in \mathcal{S}_{l k}} q_{\ell i} \tau_{c} \beta_{l \ell i}+\sum_{\ell \in \Phi i=1} \sum_{\ell i} \rho_{l \ell i}+\sigma^{2}} .
$$

Proof: It follows from applying standard LMMSE techniques [21, Ch. 12] to the problem at hand as in [17].

\section{Achievable Rates with MRC}

To detect the UL data, MRC is employed such that the combining vector is defined as $\mathbf{v}_{l l k}=\hat{\mathbf{h}}_{l l k} /\left(\gamma_{l l k} \sqrt{M \beta_{l l k}}\right)$. The estimates of the data symbols transmitted by $\mathrm{UE}_{l k}$ are obtained at $\mathrm{BS}_{l}$ as $\hat{\mathbf{s}}_{l k}^{T}=\mathbf{v}_{l l k}^{H} \mathbf{Y}_{l}$. Notice that since the pilot symbols are known at the BS, in principle, they can be subtracted from $\hat{\mathbf{s}}_{l k}$ (see second term in the right hand side of (1)) to increase the effective SINR. However, due to imperfect channel knowledge, this subtraction of pilot symbols cannot be done perfectly. In the following lemma, an ergodic achievable rate defined as a lower bound on the ergodic capacity is given considering the cases where either no pilot subtraction or perfect pilot subtraction is implemented.

Lemma 2. An ergodic achievable rate for $\mathrm{UE}_{l k}$ using $M R C$ detection is given by

$$
\mathrm{R}_{l k}=\log _{2}(1+\underbrace{\frac{M \rho_{l k} q_{l k} \beta_{l l k}^{2}}{D_{l k}}}_{=\mathrm{SINR}_{l k}})
$$

where $\mathrm{SINR}_{l k}$ is the effective SINR of $\mathrm{UE}_{l k}$, and $D_{l k}$ is the equivalent interference plus noise term described by a posynomial function ${ }^{2}$ with respect to the pilot and data powers, as show in (7) at the top of the page. Note that the variable $a_{\ell i}$ is used to characterize the case in which the pilot symbols are perfectly subtracted from the data estimates as

$$
a_{\ell i}= \begin{cases}q_{\ell i} & \text { with no pilot subtraction } \\ 0 & \text { with perfect pilot subtraction. }\end{cases}
$$

\footnotetext{
${ }^{2}$ Let $\mathbf{x}=\left[x_{1}, \ldots, x_{n}\right]^{T} \in \mathbb{R}_{+}^{n}$ where $\mathbb{R}_{+}^{n}$ is the set of $n$ dimensional vectors with positive entries, that is, $x_{j}>0 \forall j \in\{1, \ldots, n\}$. A function $f: \mathbb{R}_{+}^{n} \rightarrow \mathbb{R}$ is a posynomial if it can be expressed as $f(\mathbf{x})=\sum_{b=1}^{B} c_{b} \prod_{j=1}^{n} x_{j}^{e_{j, b}}$, for some positive integer $B$, constants $c_{b}>0$, and exponents $e_{j, b} \in \mathbb{R}$ for $j \in\{1, \ldots, n\}$ and $b \in\{1, \ldots, B\} \quad$ [2, App. B.6], [19, Ch. 4].
} 
Proof: It follows the same approach as in [17] by applying standard lower bounds on the capacity of the equivalent SISO system given after linear processing, that is, $\left[\mathbf{v}_{l l k}^{H} \mathbf{Y}_{l}\right]_{j} \forall j \in$ $\left\{1, \ldots, \tau_{c}\right\}$ [1, Ch. 2]. In the case where the pilot symbols are perfectly subtracted, the equivalent SISO system is given by $\left[\mathbf{v}_{l l k}^{H} \mathbf{Y}_{l}\right]_{j}-\sum_{\ell \in \Phi} \sum_{i=1}^{K} \mathbf{h}_{l \ell i} \sqrt{q_{\ell i}}\left[\boldsymbol{\phi}_{\ell i}\right]_{j}$. Then the variance of the effective noise is obtained by using properties of circularly symmetric complex Gaussian vectors [17], [1, App. A].

It is worth mentioning that the case in which the pilot symbols are perfectly subtracted from the data estimates is not possible to implement in practice since the channel realizations are not perfectly known. However, the achievable rate with perfect pilot subtraction can be viewed as an upper bound on the performance that could be achieved with SP by means of more involved signal processing techniques to remove the interfering effect between pilot and data symbols, like for example, iterative decoding algorithms [13], [15].

\section{Optimal Power CONTROL With SP}

Consider the following optimization problem:

$$
\begin{array}{ll}
\underset{\left\{\rho_{l k}, q_{l k}\right\}}{\operatorname{maximize}} & f\left(\operatorname{SINR}_{l k}\right) \\
\text { subject to } & \left(\rho_{l k}+q_{l k}\right) \leq P_{\max } \text { for } \quad l \in \Phi, \\
k \in\{1, \ldots, K\},
\end{array}
$$

where $P_{\max }$ is the maximum transmission power allowed per UE, assumed to be the same for all UEs, and where $f\left(\operatorname{SINR}_{l k}\right)$ corresponds to one of the following utility functions:

(A) Max-min Fairness: $f\left(\operatorname{SINR}_{l k}\right)=\min _{l, k} \operatorname{SINR}_{l k}$

(B) Max-product of SINRs: $f\left(\operatorname{SINR}_{l k}\right)=\prod_{l \in \Phi k=1} \prod^{K} \operatorname{SINR}_{l k}$.

The max-min fairness approach (A) aims at providing maximum fairness in which all UEs have equal data rates, whereas the max-product of SINRs (B) aims at balancing the aggregated performance with fairness among individual UEs, since it also maximizes sum of $\log _{2}\left(\operatorname{SINR}_{l} k\right)$; see [2, Ch. 7] for details.

Before solving the optimization problem in (8), recall that the effective interference plus noise term $D_{l k}$ in (7), at the top of the previous page, is a posynomial function with respect to the pilot and data powers. Therefore, the optimization problem in (8) can be casted as a geometric program for which efficient solvers are available [2], [19], [20]. This is the main contribution of this paper and is done below.

\section{A. Max-min fairness}

An equivalent optimization problem to (8) for the max-min fairness case can be found by recasting it to epigraph form [19]

$$
\begin{array}{ll}
\underset{\substack{\left\{\mu \geq 0, \rho_{l k} \geq 0, q_{l k} \geq 0\right\} \\
\text { subject to }}}{\operatorname{maximize}} & \mu \\
& \operatorname{SINR}_{l k} \geq \mu, \\
& \left(\rho_{l k}+q_{l k}\right) \leq P_{\max }, \text { for } \begin{array}{l}
l \in \Phi, \\
k \in\{1, \ldots, K\},
\end{array}
\end{array}
$$

$\Leftrightarrow$

$$
\begin{array}{ll}
\underset{\left\{\mu \geq 0, \rho_{l k} \geq 0, q_{l k} \geq 0\right\}}{\operatorname{maximize}} & \mu \\
\text { subject to } & \frac{\mu D_{l k}}{M \rho_{l k} q_{l k} \beta_{l l k}^{2}} \leq 1, \\
& \frac{\rho_{l k}+q_{l k}}{P_{\max }} \leq 1, \text { for } \\
& l \in \Phi \in\{1, \ldots, K\} .
\end{array}
$$

Note that both constraints involve posynomials being lower or equal to one, which are geometric constraints.

\section{B. Max-product of SINRs}

For the max-product of SINRs an equivalent optimization problem to $(8)$ is

$$
\begin{aligned}
& \underset{\left\{\rho_{l k} \geq 0, q_{l k} \geq 0\right\}}{\operatorname{maximize}} \quad \prod_{l \in \Phi k=1}^{K} \prod_{k} \operatorname{SINR}_{l k} \\
& \text { subject to } \quad \rho_{l k}+q_{l k} \leq P_{\max }, \text { for } \begin{array}{l}
l \in \Phi, \\
k \in\{1, \ldots, K\} .
\end{array} \\
& \Leftrightarrow \\
& \underset{\left\{v_{l k} \geq 0, \rho_{l k} \geq 0, q_{l k} \geq 0\right\}}{\operatorname{maximize}} \prod_{l \in \Phi k=1}^{K} \prod_{l k} \\
& \text { subject to } \quad \frac{v_{l k} D_{l k}}{\rho_{l k} q_{l k} \beta_{l l k}^{2}} \leq 1 \text {, } \\
& \frac{\rho_{l k}+q_{l k}}{P_{\max }} \leq 1, \text { for } \quad \begin{array}{l}
l \in \Phi, \\
k \in\{1, \ldots, K\} .
\end{array}
\end{aligned}
$$

In (10), an auxiliary variable, $v_{l k}$, has been introduced to enforce an SINR constraint for each UE, that in turn, can be rewritten as a geometric constraint. Thus, the optimization problem in (10) is a geometric program.

\section{Statistical ChanNEl INVERSion POWER CONTROL}

In previous works on SP for Massive MIMO [15]-[18], heuristic power control algorithms based on statistical channel inversion have been used. This approach is explained and optimized in this section, and will serve as a benchmark. The transmission powers of pilot and data symbols from $\mathrm{UE}_{l k}$ are

$$
q_{l k}=\Delta \frac{\varrho}{\beta_{l l k}}, \quad \rho_{l k}=(1-\Delta) \frac{\varrho}{\beta_{l l k}}
$$

where $\varrho \geq 0$ is used to determined the SNR level (given by $\left.\varrho / \sigma^{2}\right)$ of all UEs, and $\Delta \in[0,1]$ indicates the proportion of power allocated between pilot and data symbols. Notice that in contrast to the strategies in Section V, here the proportion between pilot and data power $\Delta$ is set equal among all UEs, and the transmit powers are decided individually for every UE without taking the co-user channels into account.

By introducing (11) into (7), the effective SINR of $\mathrm{UE}_{l k}$ results in (12) and (13) (at the top of the next page) for no 


$$
\begin{aligned}
\operatorname{SINR}_{l k}^{\mathrm{NO}-S U B} & =M /\left(\frac{2}{\tau_{c}}+\left(M+\frac{2}{\tau_{c}}\right) \Upsilon_{\mathcal{S}_{l k}}^{(2)}+\left(\left(\frac{M}{\tau_{c}}+\frac{1}{\tau_{c}^{2}}\right) \frac{1}{\Delta}-\frac{1}{\tau_{c}^{2}}\right) \Upsilon^{(2)}+\left(1+\Upsilon_{\mathcal{S}_{l k}}^{(1)}+\frac{1}{\tau_{c} \Delta} \Upsilon^{(1)}+\frac{\sigma^{2}}{\Delta(1-\Delta) \varrho \tau_{c}}\right)\left(\Upsilon^{(1)}+\frac{\sigma^{2}}{\varrho}\right)\right) \\
\mathrm{SINR}_{l k}^{\mathrm{PERF}-S U \mathrm{~B}} & =M /\left(M \Upsilon_{\mathcal{S}_{l k}}^{(2)}+\left(\frac{M}{\tau_{c}}+\frac{1}{\tau_{c}^{2}}\right)\left(\frac{1}{\Delta}-1\right) \Upsilon^{(2)}+\left(1+\Upsilon_{\mathcal{S}_{l k}}^{(1)}+\frac{1}{\tau_{c}}\left(\frac{1}{\Delta}-1\right) \Upsilon^{(1)}+\frac{\sigma^{2}}{\Delta \varrho \tau_{c}}\right)\left(\Upsilon^{(1)}+\frac{\sigma^{2}}{\varrho(1-\Delta)}\right)\right)
\end{aligned}
$$

TABLE I: Simulation Parameters

\begin{tabular}{c|c|c} 
Parameter & Symbol & Value \\
\hline Pathloss exponent & $\alpha$ & 3.76 \\
Fixed propagation loss $(1 \mathrm{~km})$ & $\omega$ & $130 \mathrm{~dB}$ \\
Squared cell side size & - & $0.2 \mathrm{~km}$ \\
Noise power & $\sigma^{2} B_{\mathrm{W}}$ & $-96.9 \mathrm{dBm}$ \\
\hline
\end{tabular}

pilot subtraction and perfect pilot subtraction, respectively. The terms

$$
\Upsilon_{\mathcal{S}_{l k}^{(l)}}^{\left({ }_{l}\right.}=\sum_{(\ell, i) \in \mathcal{S}_{l k} \backslash(l, k)}\left(\frac{\beta_{l \ell i}}{\beta_{\ell \ell i}}\right)^{\iota}, \quad \text { and } \quad \Upsilon^{(\iota)}=\sum_{\ell \in \Phi} \sum_{i=1}^{K}\left(\frac{\beta_{l \ell i}}{\beta_{\ell \ell i}}\right)^{\iota} \text {, }
$$

with $\iota \in\{1,2\}$, represent the effect of large-scale fading coefficients. Notice that the denominator of the effective SINR of $\mathrm{UE}_{l k}$ is a convex function of $\Delta \in[0,1]$ in both of the cases with no pilot subtraction and perfect pilot subtraction. Thus, the proportion between pilot and data power can be optimized efficiently to maximize the SE of the network, as long as the total transmit power per UE is predetermined.

\section{NUMERICAL RESULTS}

To provide insights into the optimal power control with SP, numerical results are given considering 8 square cells where the BSs are located at their center, and 8 UEs are dropped at uniformly random positions within each cell. ${ }^{3}$ To avoid edge effects, the wraparound technique is used. To have the same operating point for the power control strategies in Sections V-VI, $P_{\max }=\varrho /\left(\min _{l k} \beta_{l l k}\right)$ so that the UE with the lowest average channel gain can have the same SNR with all power control strategies implemented. The largescale fading coefficient is modeled as $\beta_{l l k}=\omega^{-1} d_{l l k}^{-\alpha}$ where $d_{l l k}$ is the distance between $\mathrm{BS}_{l}$ and $\mathrm{UE}_{l k}$ (measured in $\mathrm{km}), \alpha$ is the pathloss exponent, and $\omega$ is the pathloss at a reference distance of $1 \mathrm{~km}$ to account for other propagation losses like wall penetration. The main parameters used for the numerical results are given in Table I. For the implementation of statistical channel inversion power control, $\Delta$ is optimized to maximize the sum SE in the whole network.

Fig. 2 depicts the average sum SE per cell versus the number of BS antennas. The max-product of SINRs gives the highest sum SE, as expected, followed by max-min fairness and statistical channel inversion. Notice that the sum SE difference

\footnotetext{
${ }^{3}$ Note that a minimum distance of $10 \mathrm{~m}$ between UEs and BSs is enforced.
}

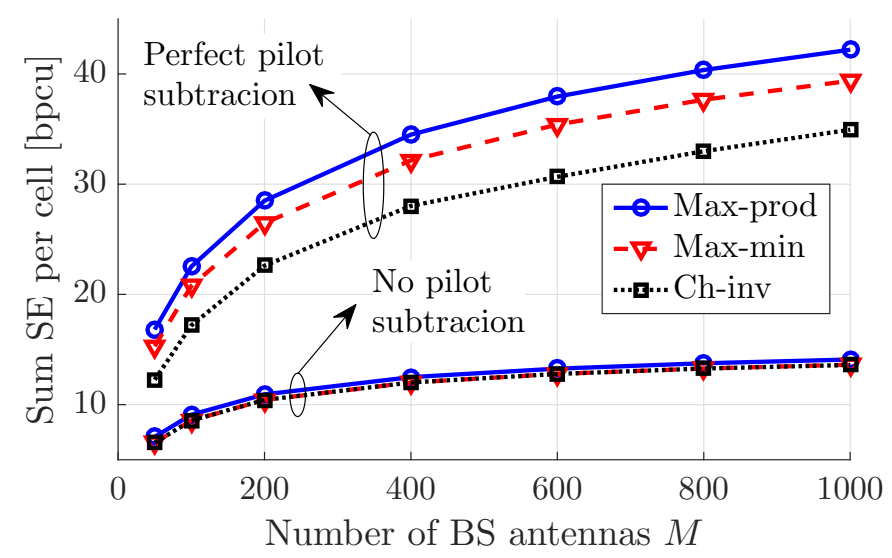

Fig. 2: Sum SE per cell [bpcu] versus $M$ for $K=8, \tau_{c}=32$ and $\varrho / \sigma^{2}=0[\mathrm{~dB}]$.

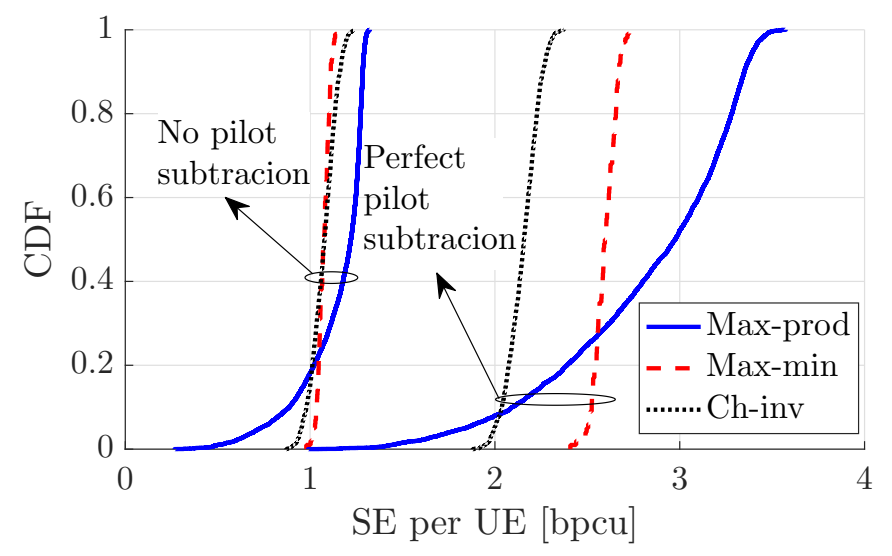

Fig. 3: Cumulative density function of the SE per UE for $M=100, K=8, \tau_{c}=32$ and $\varrho / \sigma^{2}=0[\mathrm{~dB}]$.

between the power control strategies is more pronounced for the case of perfect pilot subtraction. Thus, the use of geometric optimization strategies with polynomial complexity, like the ones in in Section V, yield more benefits when the interference from pilot symbols is reduced before data detection.

Fig. 3 shows the empirical cumulative distribution function (CDF) of the SE per UE in the system, where the randomness is due to different UE locations. Here, the tradeoff between better aggregated performance and fairness can be seen by examining the tails of the CDFs. For low percentile values, 


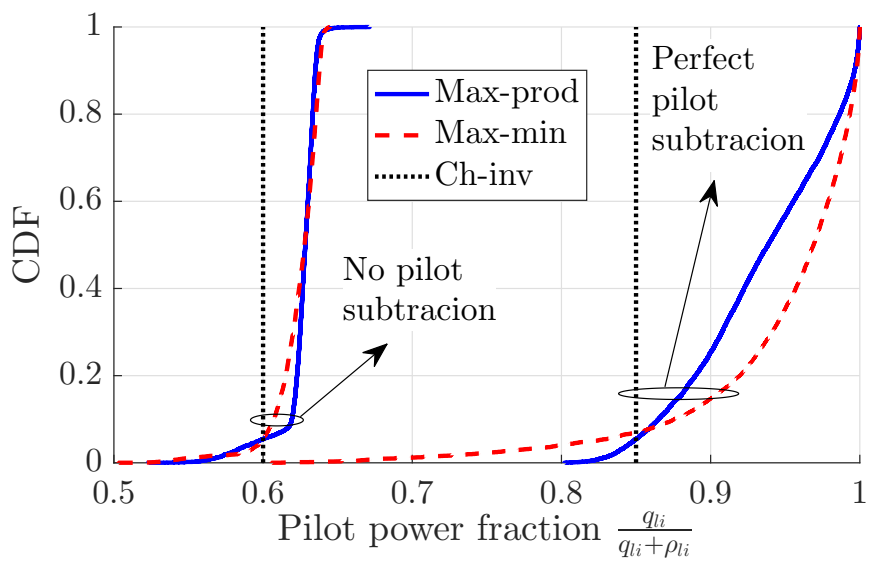

Fig. 4: Cumulative density function of the pilot power fraction per UE for $M=100, K=8, \tau_{c}=32$ and $\varrho / \sigma^{2}=0[\mathrm{~dB}]$.

max-min fairness offers higher SE followed by channel inversion and max-product of SINRs, which means, higher SE for the UEs with poor channel conditions. On the other hand, the max-product of SINRs allows for higher SE at higher percentiles where UEs with good channel conditions get better performance, and in turn, the total SE is increased. In addition, it can be seen that the benefit of optimizing the powers by using the strategies in Section $\mathrm{V}$ is more pronounced when the pilot symbols are subtracted perfectly.

Fig. 4 depicts the empirical CDF of the fraction of power that a UE allocates to pilot symbols. Here, the majority of UEs uses between $60 \%$ and $70 \%$ of the power for pilot symbols with no pilot subtraction and more than $80 \%$ with perfect pilot subtraction. Thus, with SP and MRC, the largest portion of power should be allocated to pilot symbols. This indicates that the interference from data symbols is the main source of performance degradation and the optimal power control aims at reducing the data power to mitigate this effect. Notice that the proportion between pilot and data power does not change significantly between power control strategies for the no pilot subtraction case, whereas, it has shaper differences with perfect pilot subtraction. This is in line with the previous results suggesting that when the interference from pilot symbols is reduced, a global optimization of the UE powers is able to provide appreciable SE gains.

\section{CONCLUSIONS}

This papers analyzes the optimal power control strategies for the UL of a multicell Massive MIMO system with SP and MRC detection. The results show that the max-product of SINRs and max-min fairness optimization problems for the power control with SP, can be casted as geometric programs for which efficient solvers exist. The numerical results show that appreciable SE gains from performing optimal power control over the whole network are obtained when the interference from pilot symbols is reduced. The choice of power control method is that between giving high sum SE or fairness.

\section{REFERENCES}

[1] T. L. Marzetta, E. G. Larsson, H. Yang, and H. Q. Ngo, Fundamentals of Massive MIMO. Cambridge Press, 2016.

[2] E. Björnson, J. Hoydis, and L. Sanguinetti, "Massive MIMO networks: Spectral, energy, and hardware efficiency," Foundations and Trends $®$ in Signal Processing, vol. 11, no. 3-4, pp. 154-655, Nov. 2017.

[3] T. L. Marzetta, "Noncooperative cellular wireless with unlimited numbers of base station antennas," IEEE Trans. Wireless Commun., vol. 9, no. 11 , pp. 3590-3600, 2010.

[4] H. Huh, G. Caire, H. C. Papadopoulos, and S. A. Ramprashad, "Achieving "massive MIMO" spectral efficiency with a not-so-large number of antennas," IEEE Trans. Wireless Commun., vol. 11, no. 9, pp. 32263239, September 2012.

[5] H. Yin, D. Gesbert, M. Filippou, and Y. Liu, "A coordinated approach to channel estimation in large-scale multiple-antenna systems," IEEE J. Sel. Areas Commun., vol. 31, no. 2, pp. 264-273, February 2013.

[6] E. Björnson, J. Hoydis, and L. Sanguinetti, "Massive MIMO has unlimited capacity," IEEE Trans. Wireless Commun., vol. 17, no. 1, pp. 574-590, Jan 2018.

[7] Y. Li, Y.-H. Nam, B. L. Ng, and J. Zhang, "A non-asymptotic throughput for massive MIMO cellular uplink with pilot reuse," in Proc. IEEE GLOBECOM, Dec 2012, pp. 4500-4504.

[8] E. Björnson, E. Larsson, and M. Debbah, "Massive MIMO for maximal spectral efficiency: How many users and pilots should be allocated?" IEEE Trans. Wireless Commun., vol. 15, no. 2, pp. 1293-1308, 2016.

[9] R. Mochaourab, E. Björnson, and M. Bengtsson, "Adaptive pilot clustering in heterogeneous massive MIMO networks," IEEE Trans. Wireless Commun., vol. 15, no. 8, pp. 5555-5568, Aug 2016.

[10] P. Hoeher and F. Tufvesson, "Channel estimation with superimposed pilot sequence," in IEEE Proc. GLOBECOM, 1999, pp. 2162-2166.

[11] G. T. Zhou, M. Viberg, and T. McKelvey, "A first-order statistical method for channel estimation," IEEE Signal Process. Lett., vol. 10, no. 3, pp. 57-60, March 2003.

[12] M. Dong, L. Tong, and B. M. Sadler, "Optimal insertion of pilot symbols for transmissions over time-varying flat fading channels," IEEE Trans. Signal Process., vol. 52, no. 5, pp. 1403-1418, May 2004.

[13] S. He, J. K. Tugnait, and X. Meng, "On superimposed training for MIMO channel estimation and symbol detection," IEEE Trans. Signal Process., vol. 55, no. 6, pp. 3007-3021, June 2007.

[14] A. T. Asyhari and S. ten Brink, "Orthogonal or superimposed pilots? a rate-efficient channel estimation strategy for stationary MIMO fading channels," IEEE Trans. Wireless Commun., vol. 16, no. 5, pp. 27762789, May 2017.

[15] K. Upadhya, S. Vorobyov, and M. Vehkapera, "Superimposed pilots are superior for mitigating pilot contamination in massive MIMO," IEEE Trans. Signal Process., vol. 65, no. 11, pp. 2917-2932, 2017.

[16] K. Upadhya, S. A. Vorobyov, and M. Vehkaperä, "Downlink performance of superimposed pilots in massive MIMO systems," Submitted to IEEE Trans. Wireless Commun., 2017. [Online]. Available: https://arxiv.org/abs/1606.04476v3

[17] D. Verenzuela, E. Björnson, and L. Sanguinetti, "Spectral and energy efficiency of superimposed pilots in uplink massive MIMO," Submitted to IEEE Trans. Wireless Commun., 2017. [Online]. Available: https://arxiv.org/abs/1709.07722

[18] _ - "Joint UL and DL spectral efficiency optimization of superimposed pilots in massive MIMO," in IEEE Globecom Wkshps., Dec 2017, pp. 1-7.

[19] S. P. Boyd and L. Vandenberghe, Convex Optimization. Cambridge University Press, google-Books-ID: $\mathrm{mYm} 0 \mathrm{bLd} 3 \mathrm{fcoC}$.

[20] S. Boyd, S.-J. Kim, L. Vandenberghe, and A. Hassibi, "A tutorial on geometric programming," vol. 8, no. 1, pp. 67-127. [Online]. Available: http://www.springerlink.com/index/9941458381562w82.pdf

[21] S. M. Kay, Fundamentals of Statistical Signal Processing: Estimation Theory. Prentice Hall, 1993. 